\title{
Short- and long-term outcomes following laparoscopic palliative resection for patients with incurable, asymptomatic stage IV colorectal cancer: A multicenter study in Japan
}

\author{
Tomonori Akagi ${ }^{1)}$, Masafumi Inomata ${ }^{1)}$, Suguru Hasegawa²), Yousuke Kinjo ${ }^{2)}$, Masaaki Ito $^{3)}$, \\ Yosuke Fukunaga $^{4)}$, Akiyoshi Kanazawa ${ }^{5}$, Hitoshi Idani ${ }^{6}$, Seiichiro Yamamoto ${ }^{7}$, \\ Koki Otsuka ${ }^{8}$, Shungo Endo", Masahiko Watanabe ${ }^{10)}$ and \\ Japan Society of Laparoscopic Colorectal Surgery \\ 1) Department of Gastroenterological \& Pediatric Surgery, Oita University Faculty of Medicine, Oita, Japan \\ 2) Department of Surgery, Kyoto University, Kyoto, Japan \\ 3) Department of Colorectal and Pelvic Surgery, National Cancer Center Hospital East, Chiba, Japan \\ 4) Department of Gastroenterological Surgery, Cancer Institute Hospital, Tokyo, Japan \\ 5) Department of Gastroenterology, Osaka Red Cross Hospital, Osaka, Japan \\ 6) Department of Surgery, Fukuyama City Hospital, Fukuyama, Japan \\ 7) Division of Colorectal Surgery, National Cancer Center Hospital, Tokyo, Japan \\ 8) Department of Surgery, Iwate Medical University, Iwate, Japan \\ 9) Department of Surgery, Showa University School of Medicine, Yokohama Northern Hospital, Yokohama, Japan \\ 10) Department of Surgery, Kitasato University, Sagamihara, Japan
}

\begin{abstract}
:
Objective: This retrospective multicenter study compared short- and long-term results between Japanese patients with asymptomatic stage IV colorectal cancer who underwent palliative laparoscopic surgery (LS) versus those who underwent conventional open surgery (OS). Methods: Among 968 patients treated for stage IV colorectal cancer from January 2006 to December 2007 in 41 surgical units that were participating in the Japan Society of Laparoscopic Colorectal Surgery group, we studied 398 patients who received palliative resection of their asymptomatic primary colorectal tumor. Results: We analyzed data from patients undergoing LS (LS group, $\mathrm{n}=106)$ and OS (OS group, $\mathrm{n}=292$ ). Fourteen (13.2\%) LS group patients were converted to OS. Although the differences between groups for postoperative complications were not significant, the mean time to solid food intake and postoperative length of hospital stay for the LS group were significantly shorter than those for the OS group ( 2 vs. 3 days, $\mathrm{p}<0.0001 ; 13$ vs. 16 days, $\mathrm{p}<0.0001$, respectively). The LS group patients experienced a longer median survival time than that of the OS group ( 24.5 vs. 23.9 months, $p=0.0357$ ). Conclusions: Laparoscopic palliative resection (LS) offers advantages for short-term outcomes and no disadvantages for long-term outcomes. The use of laparoscopic procedures to treat asymptomatic, incurable stage IV colorectal cancer appears to be acceptable.
\end{abstract}

Keywords:

multicenter study, laparoscopic palliative resection, incurable, asymptomatic, colorectal cancer

J Anus Rectum Colon 2017; 1(4): 125-130

\section{Introduction}

At their initial diagnoses, around $20 \%$ of patients in the United States (USA) have distant metastatic disease, and the 5-year survival rate for stage IV disease is only $13 \%{ }^{1}$. Although some controversy remains around the role of resection of the primary growth followed by systemic chemotherapy for patients with incurable stage IV colorectal cancer, 
most of these patients with incurable synchronous metastases undergo palliative resection of the primary growth, because several reports demonstrated improved long-term effects of palliative primary resection for this disease $\mathrm{e}^{2-5)}$. We previously reported the usefulness of palliative primary resection of symptomatic, incurable stage IV colorectal cancer by laparoscopic surgery (LS) compared with open surgery $(\mathrm{OS})^{6}$. To the best of our knowledge, although several reports have already evaluated the differences in clinical outcomes between LS and OS treatments for patients with incurable stage IV colorectal cancer, no studies have evaluated outcomes in terms of the presence or absence of symptoms. Generally, most surgeons consider that palliative resection of a primary colorectal tumor without symptoms might not be more difficult than that of a tumor with symptoms; however, there are few reports about and there is little reliable evidence on the surgical approach in terms of symptoms related to primary tumors.

A large, retrospective analysis was undertaken by the Japan Society of Laparoscopic Colorectal Surgery (JSLCS) to elucidate the efficacy of LS in patients with primary colorectal cancer and incurable metastases who did or did not have symptoms related to the tumor ${ }^{7)}$. We conducted a subgroup analysis of data from the JSLCS analysis to determine short- and long-term outcomes of laparoscopic palliative resection (LS) in patients with incurable but asymptomatic stage IV colorectal cancer.

Since the results of large randomized trials were published, demonstrating the safety and equivalency of LS to OS, LS has been accepted as a valid option for patients with colorectal cancer $^{8-12)}$. LS was shown to contribute to improved early postoperative recovery, with no disadvantages to cancer-related survival. These benefits might play an important role in patients with stage IV colorectal cancer. However, few reports have addressed the role of LS in patients with stage IV colorectal cancer.

Therefore, we analyzed the short- and long-term outcomes of palliative laparoscopic resection of primary colorectal cancers for patients with incurable, asymptomatic stage IV colorectal cancer. Perioperative and long-term outcomes were compared between the LS group and the OS group in the JSLCS analysis, to assess the feasibility of these proce- dures from technical and oncological viewpoints.

\section{Methods}

This multicenter, retrospective study initiated by 41 member institutions of the JSLCS was performed to compare the short- and long-term outcomes of patients with stage IV colorectal cancer undergoing palliative LS with those of patients undergoing conventional OS. The research ethics committees of all participating institutions approved this study. Demographic and clinicopathological data were collected retrospectively from consecutive patients as follows: operating time, blood loss, conversion to OS, intraoperative complications, residual tumor sites, tumor pathology, chemo- or radiotherapy, postoperative complications, length of hospital stay, and survival time. The participating surgeons were skilled in OS, with each having experience of $>100$ OS cases, and most (32 of 41) having performed $>100$ LSs. Four surgeons had performed $<30 \mathrm{LSs}$, and two of the four had performed only OS. Of the 998 patients enrolled between January 2006 and December 2007, 968 were eligible for inclusion, and patients undergoing emergency surgery were excluded. Figure 1 shows the study flowchart. Patients were assigned to one of two groups, the LS group and the OS group, based on the surgical approach deemed best by the surgeon. Also excluded were asymptomatic patients, those who underwent primary tumor resection along with resection of the metastatic site, and those scheduled at the time of the primary resection to undergo metastasectomy as a second operation (Figure 1).

Ultimately, data were analyzed for 106 LS group patients and 292 OS group patients. Preoperative patient comorbidity was assessed with the American Society of Anesthesiologists (ASA) fitness score ${ }^{13)}$. Patient-related data from the case report forms were entered into the study database, and shortand long-term outcomes were subsequently assessed.

\section{Definitions and symptoms}

Because we defined symptom-related criteria relating to colorectal tumor as those indicative of anemia (hemoglobin $<9 \mathrm{mg} / \mathrm{L}$ ) and intestinal stricture (inability to advance the colonoscope to the oral side due to the colorectal tumor) in

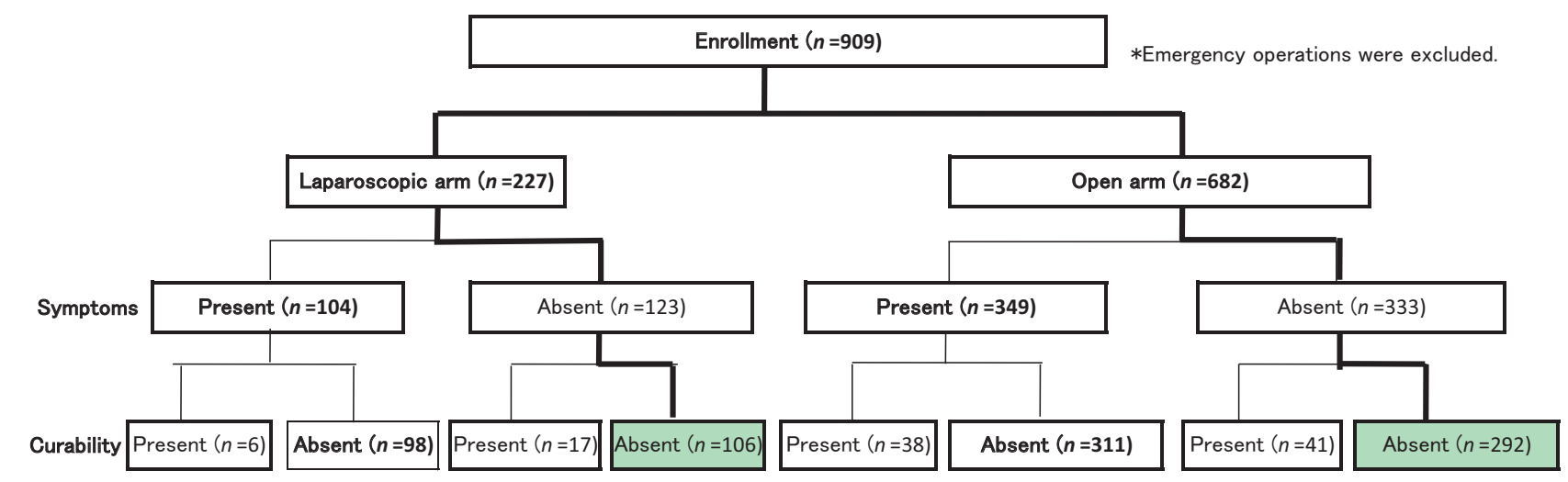

Figure 1. Flowchart of laparoscopic and open procedures for the asymptomatic, incurable stage IV colorectal cancer patients. 
Table 1. Patient Demographics.

\begin{tabular}{|c|c|c|c|}
\hline & Open $(n=292)$ & Laparoscopic $(n=106)$ & $P$ value \\
\hline Age (yrs, mean and range) & $64.1(26-95)$ & $63.7(40-87)$ & 0.905 \\
\hline Sex ratio (male:female) & $182 / 110$ & $63 / 43$ & 0.5597 \\
\hline Body mass index (kg/m, mean and range) & $22.1(13.6-42.1)$ & $22.2(17.2-28.7)$ & 0.6417 \\
\hline \multicolumn{4}{|l|}{ Procedure } \\
\hline Right colectomy & $86(29.5 \%)$ & $25(23.6 \%)$ & \\
\hline Left colectomy & $35(12.0 \%)$ & $14(4.8 \%)$ & \\
\hline Transverse colectomy & $15(5.1 \%)$ & $3(2.8 \%)$ & \\
\hline High anterior resection & $49(16.8 \%)$ & $35(33.0 \%)$ & \\
\hline Low anterior resection & $50(17.1 \%)$ & $21(19.9 \%)$ & \\
\hline AP resection & $24(8.2 \%)$ & $5(4.7 \%)$ & \\
\hline Hartmann's & $22(7.5 \%)$ & $3(2.8 \%)$ & \\
\hline Others & $11(3.8 \%)$ & 0 & \\
\hline \multicolumn{4}{|l|}{ ASA classification } \\
\hline ASA I & $147(50.3 \%)$ & $54(50.9 \%)$ & \\
\hline ASA II & $123(42.1 \%)$ & $49(46.2 \%)$ & \\
\hline ASA III & $18(6.2 \%)$ & $2(1.9 \%)$ & \\
\hline ASA IV & 0 & 0 & \\
\hline Not available & $4(1.4 \%)$ & $1(0.9 \%)$ & \\
\hline Prior abdominal surgery $(\%)$ & $62(21.2 \%)$ & $32(30.2 \%)$ & 0.862 \\
\hline \multicolumn{4}{|l|}{ Incurable metastasis } \\
\hline Liver & $212(72.6 \%)$ & $67(63.2 \%)$ & \\
\hline Lung & $80(27.4 \%)$ & $33(31.1 \%)$ & \\
\hline Peritoneum & $80(27.4 \%)$ & $5(4.7 \%)$ & \\
\hline Lymph node & $58(19.9 \%)$ & $15(14.2 \%)$ & \\
\hline Local & $2(0.7 \%)$ & $1(0.9 \%)$ & \\
\hline Others & $6(2.1 \%)$ & $5(4.7 \%)$ & \\
\hline \multicolumn{4}{|l|}{ Pathological findings } \\
\hline Depth & & & 0.420 \\
\hline $\mathrm{T} 2 / 3 / 4 \mathrm{a}[\mathrm{MP} \mathrm{SE}(\mathrm{A})]$ & 259 & 97 & \\
\hline T4b [SI (AI)] & 33 & 9 & \\
\hline pN stage & & & $\underline{0.002}$ \\
\hline Positive & 259 & 81 & \\
\hline Negative & 33 & 25 & \\
\hline
\end{tabular}

$A S A$, American Society of Anesthesiologists; $A, A I, p N$, node stage; $S E$, tumor with serosal invasion; $S I$, tumor with adjacent organ invasion

our previous study ${ }^{6}$, we defined asymptomatic eligible patients as those who, at a minimum, did not present with tumor-related anemia and intestinal stricture.

\section{Statistical analyses}

Data were compared between the LS and OS groups. We used the Chi square test to analyze differences in categorical variables including such clinicopathological factors as postoperative length of hospital stay and postoperative complications, whereas Fisher's exact test and the Student $t$-test were used as needed to analyze differences in continuous variables. We used the Kaplan-Meier method to plot overall survival curves as measured from the day of surgery and the log-rank test for comparisons. Statistical significance was considered at a $p$-value $<0.05$. We used JMP software (SAS Institute Inc., Cary, NC, USA) for all statistical analyses.

\section{Results}

\section{Demographics and surgical procedures}

As palliative surgery for asymptomatic, incurable stage IV tumors, colorectal OS was carried out in 292 patients, whereas 106 patients received colorectal laparoscopic resection. Patient demographic data are summarized in Table 1. Excluded from this study were patients who underwent emergency surgery and those with stage IV disease who could not undergo resection (i.e., those in whom palliative bypass or a proximal diverting ostomy were performed). In both groups, metastases to the liver were the most commonly incurable metastatic sites, with metastases to the lung being the second most commonly incurable sites. Resection procedures and ASA classification were not significantly different between the LS and OS groups. The number of patients with pathologically positive nodes was higher for the OS group than for the LS group but not significantly so. 
Table 2. Results of Open and Laparoscopic Techniques.

\begin{tabular}{|c|c|c|c|}
\hline & Open $(n=292)$ & Laparoscopic $(n=106)$ & $P$ value \\
\hline Operative time (mean, minutes) & $188(40-615)$ & $322(63-650)$ & $\leq 0.0001$ \\
\hline Estimated blood loss (mean, ccs) & $265.15(4-2890)$ & $119.5(0-3220)$ & $\underline{0.001}$ \\
\hline Transfusions & 29 & 7 & $\underline{0.031}$ \\
\hline Conversion & - & $14(13.2 \%)$ & \\
\hline Time to solid food intake (mean, days) & $3.6(1-42)$ & $1.9(1-20)$ & $\leq 0.0001$ \\
\hline Postoperative length of stay (mean, days) & $20.1(5-142)$ & $18(6-77)$ & $\leq 0.0001$ \\
\hline Mortality & 2 & 0 & 0.393 \\
\hline \multicolumn{4}{|l|}{ Morbidity } \\
\hline Anastomotic leakage & 6 & 3 & 0.646 \\
\hline Abscess & 2 & 1 & 0.792 \\
\hline Wound infection & 18 & 8 & 0.687 \\
\hline Bacterial enteritis & 1 & 0 & 0.546 \\
\hline Ileus & 21 & 5 & 0.377 \\
\hline Others & 15 & 2 & 0.156 \\
\hline Total & $63(21.6 \%)$ & $19(17.9 \%)$ & 0.426 \\
\hline \multicolumn{4}{|l|}{ Blood analysis } \\
\hline \multicolumn{4}{|l|}{ Leukocyte count } \\
\hline Day $1\left(\right.$ mean, $\left.\times 10^{9} / \mathrm{L}\right)$ & $18,600(2,900-23,200)$ & $8,810(1,200-20,100)$ & $\underline{0.002}$ \\
\hline \multicolumn{4}{|l|}{ C-reactive protein } \\
\hline Day 1 (mean, mg/dL) & $7.3(0.06-26.2)$ & $6.7(1.1-26.8)$ & 0.095 \\
\hline
\end{tabular}

Table 3. Postoperative Chemotherapy.

\begin{tabular}{lccc}
\hline & Open $(n=292)$ & Laparoscopic $(n=106)$ & $P$ value \\
\hline Chemotherapy performed & $247(84.6 \%)$ & $99(93.4 \%)$ & $\underline{0.02}$ \\
Treatment regimen (1st line) & & & \\
FOLFOX & $119(48.2 \%)$ & $54(59.0 \%)$ & \\
FOLFIRI & $14(5.7 \%)$ & $7(7.1 \%)$ & \\
SOX & 0 & $2(2.0 \%)$ & \\
IFL & $6(2.4 \%)$ & $1(1.0 \%)$ & \\
LV/5FU & $4(1.6 \%)$ & $2(2.0 \%)$ & \\
UFT/Uzel & $25(10.1 \%)$ & $10(10.1 \%)$ & \\
TS-1 & $13(5.3 \%)$ & $2(2.0 \%)$ & \\
FOLFOX+molecular targeted agent & $17(6.9 \%)$ & $5(5.1 \%)$ & \\
FOLFIRI+molecular targeted agent & $2(0.81 \%)$ & 0 & \\
SOX+molecular targeted agent & $1(0.4 \%)$ & 0.68 \\
Others & $46(18.6 \%)$ & $16(16.2 \%)$ & \\
Interval from operation to chemotherapy (mean, days) & $28(8-384)$ & $27(9-772)$ & \\
\end{tabular}

\section{Perioperative outcomes of $O S$ and $L S$}

Short-term outcomes and pathological findings of the two groups are listed in Table 2. Fourteen (13.2\%) patients in the LS group were converted to OS for reasons including tumor fixation attributed to primary progression in nine patients, massive peritoneal dissemination in two, adhesions in two, and remarkable lymph node metastasis in one. There were also significant differences between the OS and LS groups for the mean values of operative time (188 vs. 322 min), estimated blood loss (265.15 vs. $\left.119.5 \mathrm{~cm}^{3}\right)$, time to solid intake (3.6 vs. 1.9 days), postoperative length of hospital stay (20.1 vs. 18 days), and leukocyte count on day 1 $\left(18.6\right.$ vs. $\left.8.81 \times 10^{9} / \mathrm{L}\right)$. Postoperative complications were present in $63(21.6 \%)$ OS group patients and in $19(17.9 \%)$
LS group patients, and overall complications were not significantly different between the two groups.

\section{Postoperative chemotherapy and rates of overall survival following $\mathrm{LS}$ and $\mathrm{OS}$}

Postoperative treatment regimens are summarized in Table 3. Postoperative chemotherapy was administered in 247 (84.6\%) OS group patients and in 99 (93.4\%) LS group patients. A significantly greater number of patients underwent chemotherapy in the LS group than in the OS group. Patients in the OS group were treated with FOLFOX $(n=119)$, FOLFIRI $(n=14)$, SOX $(n=0)$, IFL $(n=6)$, LV/5FU $(n=4)$, UFT/Uzel ( $n=25)$, TS-1 ( $n=13)$, FOLFOX + a molecular targeted agent $(\mathrm{n}=17)$, FOLFIRI + a molecular targeted agent $(n=2)$, and SOX + a molecular targeted agent $(n=1)$, whereas 


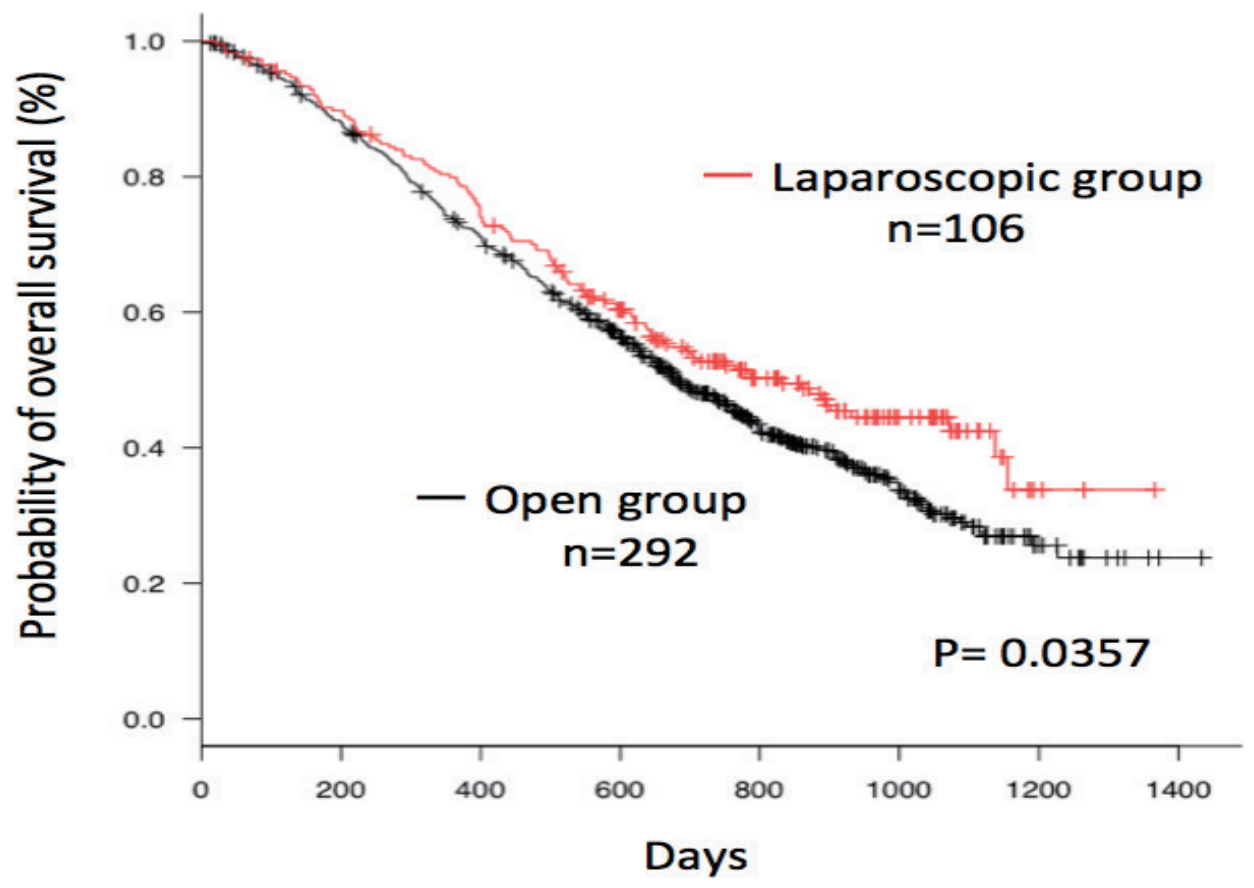

Figure 2. Kaplan-Meier estimates of overall survival of the stage IV colorectal cancer patients in the laparoscopic surgery group and open surgery group.

patients in the LS group were treated with FOLFOX $(\mathrm{n}=54)$, FOLFIRI $(n=7), \operatorname{SOX}(n=2), \operatorname{IFL}(n=1), \operatorname{LV} / 5 F U(n=2)$, UFT/Uzel $(n=10)$, TS- $1(n=2)$, and FOLFOX + a molecular targeted agent $(n=5)$. There were no significant differences between the two groups in the chemotherapy treatments given and the time from surgery to initiating postoperative chemotherapy. The respective median survival times and rates of 2-year survival were 24.5 months and $62.8 \%$ for the LS group and 23.9 months and $50.0 \%$ for the OS group. Overall survival was significantly longer for the LS group compared with that for the OS group $(p=0.0357)$ (Figure 2).

\section{Discussion}

In Japan, as in the USA, around $20 \%$ of patients with colorectal cancer also have metastatic disease at their initial diagnosis ${ }^{1,14}$. Despite major progress in systemic chemotherapy that offers new therapeutic options and improves the overall survival of these patients, the 5-year overall survival rates in Japan and the USA are still only $18.8 \%$ and $13 \%$, respectively ${ }^{1,14)}$. Thus, the quality of life of these patients with a very poor prognosis needs to be sustained, and chemotherapy must be smoothly initiated and carried out. Whether it is best to resect the primary growth first and then perform systemic chemotherapy for stage IV colorectal cancer patients remains unclear. However, palliative resection of the primary growth is performed in most of these patients, on the basis of several reports that have shown improvement in the long-term effects of palliative bowel resection ${ }^{3-5}$. Despite the lack of reliable evidence demonstrating the benefit for laparoscopic primary resection for patients with stage IV colorectal cancer, the number of laparoscopic resections has been rising in recent years. In this study, we evaluated the safety and efficacy of LS for patients with asymptomatic, incurable stage IV colorectal cancer, after previously evaluating these factors in symptomatic patients with incurable stage IV disease ${ }^{6}$. Although there are limitations in fully describing symptom criteria, we defined asymptomatic to mean no presentation of anemia or intestinal stricture, as detailed above.

In general, the use of laparoscopy in patients with curable colorectal cancer is accepted, because of the significant perioperative benefits to patients, as indicated by several randomized trials ${ }^{8-12}$. Unfortunately, whether laparoscopic procedures are best for patients with stage IV colorectal cancer, regardless of their symptoms, is unclear. To the best of our knowledge, although several previous reports evaluated the differences in clinical outcomes between LS and OS treatments for patients with incurable stage IV colorectal cancer, symptoms were not considered; therefore, we believe this is the first study to evaluate short- and long-term outcomes following palliative LS of the primary tumor for patients with asymptomatic, incurable stage IV colorectal cancer compared with those for OS.

Short-term outcomes appeared to be more favorable for the patients who underwent palliative LS than for those who underwent conventional OS. The postoperative length of hospital stay was shorter for the LS group than that for the OS group $(p=0.024)$. Although 63 (21.6\%) OS group patients and 19 (17.9\%) LS group patients experienced postoperative complications, the difference between groups was not significantly different. The beneficial effects of palliative LS on short-term outcomes found in present study were almost equivalent to those reported in previous large randomized 
trials of laparoscopic curative resection of colorectal cancers.

More patients in the LS group (99, 93.4\%) underwent postoperative chemotherapy than those in the OS group $(247,84.6 \%)$, and the difference was significant $(p=0.02)$. Postoperative chemotherapy was not performed in either group due to comorbidities, advanced age ( $>75$ years), and the patient's wish. The time from surgery to the beginning postoperative chemotherapy was not different between the two groups. Almost all patients in this study were treated with new forms of systemic chemotherapy comprising modern combination regimens, and the between-group difference in regimens was not statistically significant.

The respective median survival times and rates of 2-year survival were 24.5 months and $60 \%$ for the LS group and 23.9 months and $50.0 \%$ for the OS group. The overall survival rate was significantly longer for the LS group than for the OS group $(p=0.007)$. So, far, the prognostic factors for incurable stage IV cancer shown by several studies include ASA classification, performance status, white blood cell count, chemotherapy regimen, and the number of metastatic organs ${ }^{5,15-17)}$. The differences in ASA classifications, chemotherapy regimens, and the number of metastatic organs between the groups in the present study were not significant. We think that there are two possible reasons for the better overall survival of the LS versus that of the OS group. First, the rate of pathologically positive nodes was less for the LS group than that for the OS group $(p=0.002)$, and, second, more than twice as many LS group patients underwent postoperative chemotherapy than OS group patients $(p=0.02)$.

The present study showed an association between laparoscopic palliative resection (LS) and improved early postoperative recovery, along with no disadvantages for long-term outcomes in comparison with open resection. In this retrospective cohort study, surgeons dictated the choice of operative procedure, and, thus, the most important limitation of a study of this type is selection bias. A better method to evaluate the efficacy of LS versus OS is the randomized, controlled study. To establish treatment choices for patients with asymptomatic, incurable stage IV colorectal cancer, additional studies should be performed to assess the usefulness of LS compared to OS in improving surgical outcomes.

\section{Conflicts of Interest}

There are no conflicts of interest.

\section{References}

1. Siegel RL, Miller KD, Jemal A. Cancer statistics, 2016. CA Cancer J Clin. 2016 Jan-Feb; 66(1): 7-30.

2. Shida D, Hamaguchi T, Ochiai H, et al. Prognostic impact of palliative primary tumor resection for unresectable stage 4 colorectal cancer: using a propensity score analysis. Ann Surg Oncol. 2016 Oct; 23(11): 3602-8.

3. 't Lam-Boer J, Van der Geest LG, Verhoef C, et al. Palliative re- section of the primary tumor is associated with improved overall survival in incurable stage IV colorectal cancer: a nationwide population-based propensity-score adjusted study in the Netherlands. Int J Cancer. 2016 Nov; 139(9): 2082-94.

4. Yang Q, Liao F, Huang Y, et al. Longterm effects of palliative local treatment of incurable metastatic lesions in colorectal cancer patients. Oncotarget. 2016 Apr; 7(15): 21034-45.

5. Ruo L, Gougoutas C, Paty PB, et al. Elective bowel resection for incurable stage IV colorectal cancer: prognostic variables for asymptomatic patients. J Am Coll Surg. 2003 May; 196(5): 722-8.

6. Akagi $\mathrm{T}$, Inomata $\mathrm{M}$, Kitano $\mathrm{S}$, et al. Multicenter study of shortand long-term outcomes of laparoscopic palliative resection for incurable, symptomatic stage IV colorectal cancer in Japan. J Gastrointest Surg. 2013 Apr; 17(4): 776-83.

7. Hida K, Hasegawa S, Kinjo Y, et al. Open versus laparoscopic resection of primary tumor for incurable stage IV colorectal cancer: a large multicenter consecutive patients cohort study. Ann Surg. 2012 May; 255(5): 929-34.

8. Lacy AM, Delgado S, Castells A, et al. The long-term results of a randomized clinical trial of laparoscopy-assisted versus open surgery for colon cancer. Ann Surg. 2008 July; 248(1): 1-7.

9. Clinical Outcomes of Surgical Therapy Study Group. A comparison of laparoscopically assisted and open colectomy for colon cancer. N Engl J Med. 2004 May; 350(20): 2050-9.

10. Fleshman J, Sargent DJ, Green E, et al. Laparoscopic colectomy for cancer is not inferior to open surgery based on 5-year data from the COST Study Group trial. Ann Surg. 2007 Oct; 246(4): 655-62; discussion 662-4.

11. Buunen M, Veldkamp R, Hop WC, et al. Survival after laparoscopic surgery versus open surgery for colon cancer: long-term outcome of a randomised clinical trial. Colon Cancer Laparoscopic or Open Resection Study Group. Lancet Oncol. 2009 Jan; 10(1): 44-52.

12. Guillou PJ, Quirke P, Thorpe H, et al. Short-term endpoints of conventional versus laparoscopic-assisted surgery in patients with colorectal cancer (MRC CLASICC trial): multicentre, randomised controlled trial. Lancet. 2005 May; 365(9472): 1718-26.

13. Keats AS. The ASA classification of physical status--a recapitulation. Anesthesiology. 1978 Oct; 49(4): 233-6.

14. Watanabe T, Itabashi M, Shimada $Y$, et al. Japanese Society for Cancer of the Colon and Rectum (JSCCR) Guidelines 2014 for treatment of colorectal cancer. Int J Clin Oncol. 2015 Apr; 20(2): 207-39.

15. Cook AD, Single R, McCahill LE. Surgical resection of primary tumors in patients who present with stage IV colorectal cancer: an analysis of surveillance, epidemiology, and end results data, 1988 to 2000. Ann Surg Oncol. 2005 Aug; 12(8): 637-45.

16. Stelzner S, Hellmich G, Koch R, et al. Factors predicting survival in stage IV colorectal carcinoma patients after palliative treatment: a multivariate analysis. J Surg Oncol. 2005 Mar; 89(4): 211-7.

17. Kleespies A, Fuessl KE, Seeliger H, et al. Determinants of morbidity and survival after elective non-curative resection of stage IV colon and rectal cancer. Int J Colorectal Dis. 2009 Sep; 24(9): 1097-109.

Journal of the Anus, Rectum and Colon is an Open Access article distributed under the Creative Commons Attribution-NonCommercial-NoDerivatives 4.0 International License. To view the details of this license, please visit (https://creativecommons.org/licenses/by-nc-nd/4.0/). 